\title{
AN AFFORDANCE-BASED FORMALISM FOR MODELING HUMAN-INVOLVEMENT IN COMPLEX SYSTEMS FOR PROSPECTIVE CONTROL
}

\author{
Namhun Kim \\ Design and Human Engineering \\ Ulsan National Institute of Science and Technology \\ Ulsan, 689-798, REPUBLIC OF KOREA
}

Ling Rothrock

Industrial and Manufacturing Engineering

The Pennsylvania State University

University Park, PA 16802, USA

\author{
Jaekoo Joo \\ Systems and Management Engineering \\ Inje University, Gimhae
}

Gyeongnam, 621-749, REPUBLIC OF KOREA

\author{
Richard A. Wysk \\ Industrial and Systems Engineering \\ North Carolina State University \\ Raleigh, NC 27695, USA
}

\begin{abstract}
We propose a predictive modeling framework for human-involved complex systems in which humans play controlling roles. Affordance theory provides definitions of human actions and their associated properties, and the affordance-based Finite State Automata (FSA) model is capable of mapping the nondeterministic human actions into computable components in modeling formalism. In this paper, we further investigate the role of perception in human actions and examine the representation of perceptual elements in affordance-based modeling formalism. We also propose necessary and sufficient conditions for mapping perception-based human actions into systems theory to develop a predictive modeling formalism in the context of prospective control. A driving example is used to show how to build a formal model of humaninvolved complex system for prospective control. The suggested modeling frameworks will increase the soundness and completeness of a modeling formalism as well as can be used as guide to model human activities in a complex system.
\end{abstract}

\section{INTRODUCTION}

In spite of the importance of modeling human actions, few formal modeling methodologies have been developed to formally describe human actions in complex systems. To model human actions in context of the task environment, we begin with the concept of an affordance. Gibson (1979) introduced the term affordance to define, "what is offered to an animal either for its ill or good". Affordances are what one can do, not what one must do. More specifically, we take affordance to be a property of the environment taken with respect to a human operator and it provides an opportunity for an action by the operator (either for its good or for ill) within that environment in order to achieve a particular goal or objective.

We assess affordances in the context of operator control of a system based on future events and goals. This form of control is also known as prospective control. We also use the concept that affordances are coupled with a complementary human capability to effect action. This human property is known as effectivity. Because our model assumes human control of a system in real time, we focus on the human operator's perception of the system as well as his ability to act upon the system. When considering perceptual elements in modeling a human action, it is difficult to represent the dynamic aspects of perceptions into 


\section{Kim, Joo, Rothrock and Wysk}

simple discrete model data. While perception involves complex cognitive processes, an affordance provides a naturalistic means for representing and predicting the interaction between a human and his or her surrounding environment. However, consideration of human perception is essential to account for the states of affordance and effectivity in designing a human-involved system.

Kim et al. (2008; 2010) proposed an affordance-based descriptive modeling formalism using finite state automata (FSA). However, in the paper, even though the properties of affordances and effectivities model what human action occurred in a descriptive manner, few explanations about how to increase the completeness of the system models for prescriptive uses were presented. In this paper, we further investigate the role of perceptual elements in human action and propose potential spatio-temporal necessary and sufficient conditions of a perception-based human action in the complex system. We emphasize that prospective control is a key to map human roles into systems theory.

The objective of the paper is to develop a prescriptive modeling framework for representing human involvement in complex systems for prospective control. To carry out this objective, first, we identify the role of perception in human actions to supply the basic properties of an affordance-based modeling formalism. Second, we investigate the necessary and sufficient conditions for mapping perception-based human actions into systems theory with consideration of perceptual elements that are composed of sensory information. Third, we suggest an affordance-based modeling formalism to build a perception-based predictive model of human actions using finite state automata (FSA) for prospective control. Finally, a simple driving example is illustrated to show the breadth and depth of modeling applicability.

The remainder of this paper is organized as follows: In Section 2, we present affordance theory and formal representations of affordance models. We discuss the relevant issues for developing an affordancebased FSA model including the hierarchy of perceptual properties in Section 3. In Section 4, a generic modeling framework for building an affordance-based FSA formalism is presented. Section 5 provides a sample application to illustrate the modeling methodology presented in this paper. We provide concluding remarks in Section 6.

\section{LITTERATURE REVIEW}

\subsection{Theory of Affordance in Prospective Controls}

Gibson (1979) defines an affordance as an environmental property taken with respect to an animal (person) and it provides an opportunity for an action by the animal (either for its good or for ill) within that environment in order to achieve a particular goal or objective. In perspectives of goal-directed control, an affordance, an opportunity for action, can also be considered a precondition of prospective control (PC).

An environment offers a different set of affordances that may or may not be concurrently available to multiple animals that reside within this animal environment system (AES). For instance, in a humansitting-on-a-chair system, a chair provides the affordance "is sit-able" to a person who intends to take a seat and "is-stand-on-able" to a person who wants to use it as a pedestal.

Since Gibson proposed his definition, proponents and opponents of affordance theory have offered several definitions of affordances. Key to all the definitions is the role of perception. According to Jones (2003) and Shaw (2003), the theories relating to perception fall into two categories - Direct or Indirect Perception. Theories of direct perception assume that objects and events have inherent meanings, which an animal can exploit without any cognitive effort. Jones argues that Gibson's affordance theory conforms to direct perception category (Jones 2003). On the other hand, theories that constitute indirect perception assume that objects and events have no inherent meaning, which necessitates the internal creation of meaning that must be stored by the animal. Simon's indirect perception theory falls under this category (Simon 1969). Simon argues that coupling is an artifact (rule-governed) that is mediated by symbol functions essentially involving information processing.

In addition to this, a model based on the concept of "coalition" is offered by Shaw and Turvey (1981), which suggest that perceptual organization is an activity of the ecosystem. The coalition model posits that 


\section{Kim, Joo, Rothrock and Wysk}

symmetry of constraints exists between the animal and its environment within an ecosystem. It suggests that the organization of perception and action within the ecosystem is not a mere achievement of the animal's nervous system but rather an activity of the environment. The coalition model, therefore, stipulates that successful goal-directed action depends on one's ability to perceive the behavioral possibilities of one's environment. For example, a one-meter ledge affords stepping down for an adult but not for a small child. The fundamental notion in Gibson's theory of affordances is that affordance is real property of the animal-environment system that is perceived directly toward the execution of a potential action (Turvey 1992). In other words, meaning does not originate in the observer's head, but is perceived by detecting information about affordances from the environment. Prospective control of an action is pivotal to the successful performance of both routine acts, such as navigating through a busy college campus, and highly skilled tasks, such as positioning oneself to catch a fly ball in a baseball game. In both cases, success depends on the ability to perceive the possibilities for action within the environment, so that one's movement within the environment can be appropriately coordinated.

Turvey (1992) presents a perspective on the ecological ontology of affordances with links to prospective control. Turvey bases the definition of affordance in terms of properties that represent a potential state and are not currently realized (called dispositional properties or dispositions). Dispositions occur in pairs in which some property of the environment (i.e., climb-able) is complemented by a property of the animal's capability known as an effectivity (i.e., to climb or walk on).

Affordance theory has been adopted by the user interface design community. In particular, it is considered one of the most important aspects of usability (Norman 1988). An affordance-based modeling approach is also used for designing robot controls and mimicking human actions in specific environmental situations (Arkin 1988 ; Kirlik et al. 1993).

Although the works of Gibson's and Turvey's provide a basis for formalizing how humans interact with complex system environs, they lack formal structure to model human actions with respect to system transitions.

\subsection{Formal Representation of Affordance in Systems Modeling}

The terms of affordance and effectivity can be combined together so that they incur a different property to be activated (Turvey 1992). Specifically, Turvey presents a formal definition of affordances mathematically using a juxtaposition function as follows;

Let $W_{p q}=j\left(X_{p}, Z_{q}\right)$ be a function that is composed of two different objects, $X$ and $Z$; and further $p$ and $q$ be properties of $X$ and $Z$, respectively. Then, $p$ refers to an affordance of $X$ and $q$ is the effectivity of $Z$, if and only if there exists a third property $r$ such that:

i) $W_{p q}=j\left(X_{p}, Z_{q}\right)$ possesses $r$,

ii) $W_{p q}=j\left(X_{p}, Z_{q}\right)$ possesses neither $p$ nor $q$, and

iii) Neither $X$ nor $Z$ possesses $r$, where $r$ is a joining or juxtaposition function.

For example, a person $(Z)$ can walk $(q)$, stairs $(X)$ can support something $(p)$, and they together yield climbing property $(r)$, which is a new property of the 'person-climbing-stairs' system. This formal definition can be mapped to the state transition function in the FSA. The existence of formal definition of an affordance provides a foundation that the concept of an affordance can be combined with systems theory.

Thiruvengada and Rothrock (2006) provide a computational model of Gibson's affordance based on Colored Petri Nets (CPN) within the context of a highway-exiting problem. In their computational formalism, an affordance is represented by the presence of tokens (that possesses different colors or attributes based on the occupancy of that lane) within each lane node of the highway lane (environment) CPN model. The effectivities are represented by tokens with colors or attributes (current position, speed, acceleration) corresponding to each driver within the driver (animal) CPN model. These tokens are filtered using the juxtaposition (filtering) function that is implemented as an algorithm within animalenvironment CPN model to create a set of potential actions based on prospective control. Under suitable 
circumstances, a potential action leading to the accomplishment of goal is actualized into a real action based on driver goals.

Kim et al. (2008) suggest a descriptive formalism for human-involved complex systems. In their work, the authors regard the states of the environmental system as discrete ones and consider the transitions among the states which are triggered by possible actions of animals, an ecosystem of an environment with human participation that can be represented by a finite state automaton. The theory of automata corresponds to the ecological sense of affordances for at least the following two reasons: First, an environmental system can be defined as a set of nodes and arcs which describe discrete states of the system and the transitions between states, respectively. Second, a set of transitions between states represents a set of potential properties (affordances) of the environmental system which can be triggered by certain human activities and lead to the next states. Therefore, affordance-effectivity combinations can be considered conditions for identifying possible human actions in FSA representations as shown in Figure 1.

Although the model describes the basic components of human-involved system behaviors, it is not adequate to be used to predict human activities in complex system environments. The model lacks the completeness of necessary and sufficient conditions for existence of perception-based human activity in the context of perception-based actions.

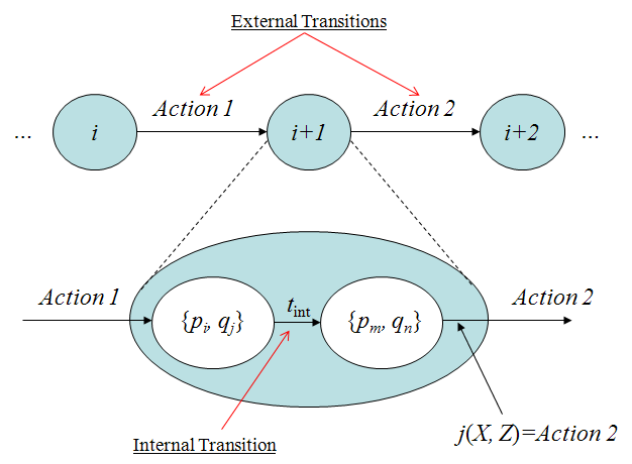

Figure1: External and internal transitions of Affordance-FSA formalism (Kim et al. 2008).

\section{AFFORDANCES FOR MODELING HUMAN-INVOLVED SYSTEMS}

In the sense of goal-directed actions, every human action has its own intent or objective. A human decision to take an action is made based on a set of information he or she receives from an environmental system. In the case of goal-directed actions, human beings make plans and assess their goals by means of information acquired by their sensory organs such as eyes, ears, skin, nose, and mouth (Gibson 1966). In other words, humans take actions based on sensory information and they actively or passively forage information from environments to generate proper plans for a specific goal (Priolli 1999).

In prospective controls, modelers are more interested in the goal-directed human actions rather than the unexpected human actions due to errors. Thus, the specific properties with which we are concerned are perception-based. The information perceived by a human can be translated into affordance-effectivity duals and a possible resulting action set in the system. When dealing with perception-based actions, we should consider affordances and effectivities as (rightly) perceived properties. In some cases, wrong perception leads toward unexpected actions. Then we may need alternative ways to correct the errors and reroute the plans or goals. For now, the existing FSA model does not consider perception errors and we only consider two cases: a specific action has occurred or has not. 


\subsection{Perception of Affordances, Effectivities, and Possible Actions}

Fajen (2005) also suggests that the properties of affordances and effectivities are higher order ones that can be perceived directly. In other words, the higher order properties can be expressed as a function of lower order properties which contains perceivable information. We propose that the outcome of the affordance can be discretized and modeled as an FSA.

Perception is the process of attaining awareness or understanding sensory information (Levine 2000). It is a complex cognitive process that enables us to recognize environmental situations or states via sensory inputs (Gibson 1966; 1982). Perception is generally defined as three different sequential processes: selection, organization, and interpretation (Huffman 2007). The decision process model is generally depicted as shown in Figure 2. As the sensory information is attained and screened by 'selection' through body sensors, the stimuli are arranged by 'organization' in a meaningful way. 'Interpretation' is the last stage of perception to attach meanings to the information. In light of perception, the output of the process modifies the animal-environment system.

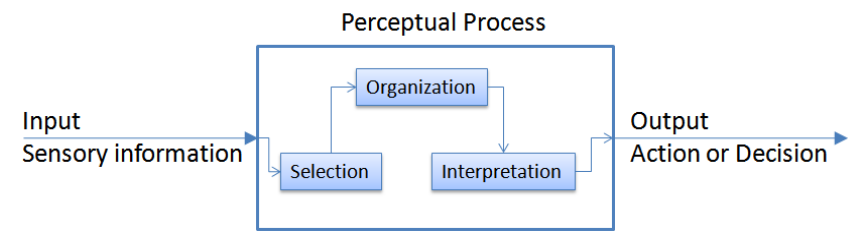

Figure 2: Decision process.

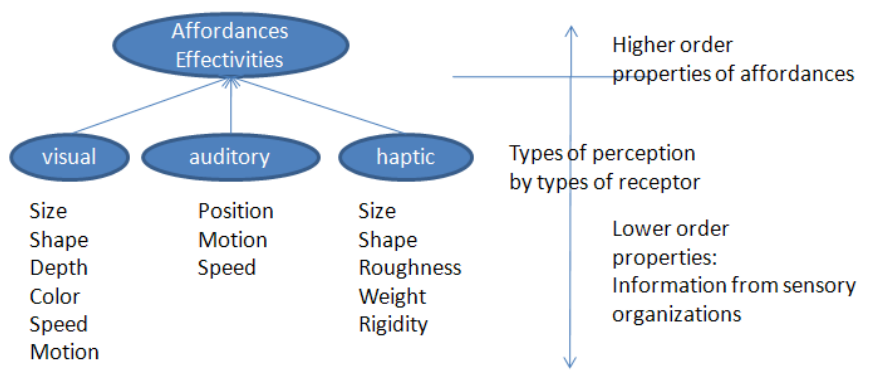

Figure 3: Hierarchy of properties in perception-based actions.

The basic information obtained from one's surroundings acts as an input for a perceptual process that comes through sensory organs. When we try to model perception-based human actions, the information that we perceive from our environs could be sensory information which are subordinate to higher order properties, affordances and effectivities. The hierarchical relationship between lower order properties and higher order properties in perception-based human actions is shown in Figure 3. We emphasize that the higher and lower order properties are only defined for perception-based and goal-directed actions. In this case, we deal with only three categories of sensory information because they possess the major properties of three dimensional features that result in the physical interactions between an animal and an environment.

Affordances and effectivities may experience complicated perceptual processes in order to be evaluated, although they are a property that is directly perceived as defined by their definitions.

Kim et al. (2008) suggest a formalism representation for human-involved complex systems. The authors defined a 6-tuple affordance-based FSA, $\boldsymbol{M}^{\text {comb }}$ (Combined model), that describes the rules of state transitions as follows; 


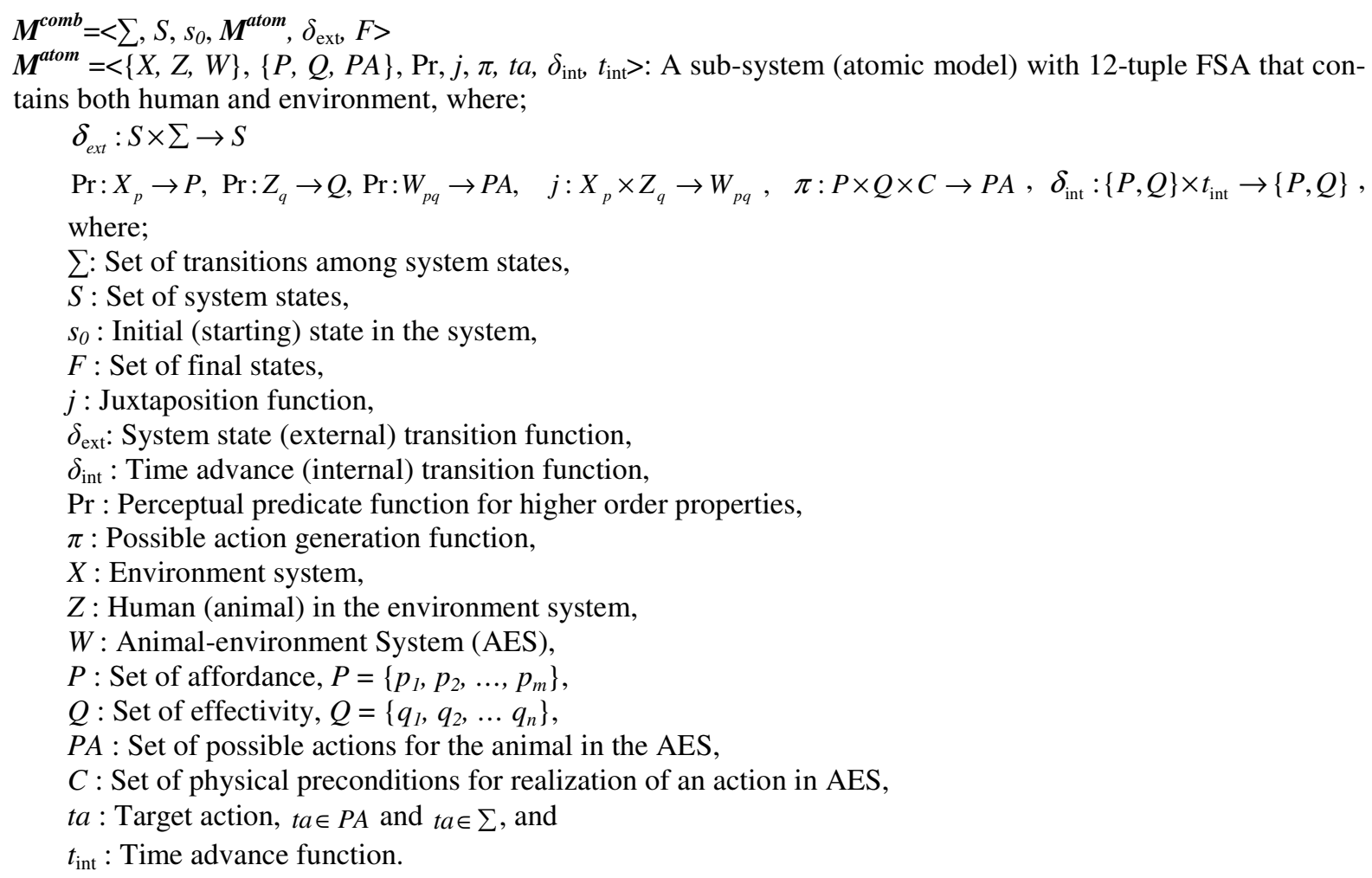

In the model suggested, the perceptual predicate function Pr acts as a perceptual process that generates higher order properties of perceived affordances $(\mathrm{P})$, effectivities $(\mathrm{Q})$, and possible actions (PA) by receiving perceivable low order property information through sensory organs in AES. The function, Pr, maps objects to higher order perceptual properties (such as affordance and effectivity) in a specific instance when a predictive human perception process is occurred. The perceptual predicate function can be further decomposed into sub functions each of which processes stimuli from different sensory organs. We will again use the example of a human-sitting-on-a-chair system. In this example, an affordance, sitability of a chair, is visually perceived by a person who has an effectivity to sit on the chair. The affordance and effectivity are higher order properties that are directly perceived by the person and they consist of a set of lower order perceptual properties such as shape and dimensions of the chair and the person.

From the definition of the perceptual decision process in Figure 2, the perceptual predicate function can be expressed as a series of functions of selection, organization, and interpretation. We suggest that in the stage of selection, the sensory inputs can be exclusively classified into three different types of lower order properties by receptors;

Pr: $W_{p q} \rightarrow$ Interpretation: Organization : Selection: $W_{p q}$, and

Selection: $W_{p q} \rightarrow \cup_{i} \varphi_{i}\left(W_{p q}\right)$, where $i=\{v, a, h\}$,

$\varphi_{v}$ : Visually perceptual predicate function for lower order properties,

$\varphi_{a}$ : Auditorily perceptual predicate function for lower order properties, and

$\varphi_{h}$ : Haptically perceptual predicate function for lower order properties.

In the same ways for affordances and effectivities, the perceptual higher order properties are represented as functions of perceptual lower order properties as follows;

$f_{\varphi}: \bigcup_{i}\left\{\varphi_{i}: W_{p q}\right\} \rightarrow P A, f_{\varphi}: \bigcup_{i}\left\{\varphi_{i}: X_{p}\right\} \rightarrow P$, and $f_{\varphi}: \bigcup_{i}\left\{\varphi_{i}: Z_{q}\right\} \rightarrow Q$

, where $f_{\varphi}$ : A function of organization and interpretation in a perceptual process and $i=\{v, a, h\}$. 


\section{Kim, Joo, Rothrock and Wysk}

Since human actions in prospective control schemas are taken based on humans' sensory information, the lower order properties should be perceivable through sensory organs either visually, auditorily, or haptically in order to be used to model perception-based and goal-directed human behaviors (Gibson 1966). When modeling human participation in systems, the perceptual higher order properties of affordances, effectivities, and possible actions can be represented as functions of the perceptual lower order properties. This makes modeling procedure more logical and complete because the lower order properties in systems are relatively easier to be found by modelers than the higher order perceptual properties.

\subsection{Necessary-Sufficient Conditions of Perception-based Actions in Systems Theory}

In Turvey's definition of affordance, $W_{p q}$ means an AES generated by juxtaposing between $X_{p}$ and $Z_{q}$, such that $W_{p q}=j\left(X_{p}, Z_{q}\right)$ (Turvey 1992). In the case of a perception-based action, the perception of $W_{p q}$ is essentially accompanied by the perception of $X_{p}$ and $Z_{q}$. By perceiving the lower order properties of $X_{p}$ and $Z_{q}$, the higher order properties of affordance and effectivity are perceived, and it leads to perception of a possible action. In short, without perception of both an affordance and an effectivity, the perception of a possible action does not exist.

In prospective controls, perception-based actions are the result of joining between affordances and effectivities that are also perceivable. The necessary condition for perception-based human actions in prospective controls is defined as Proposition 3.1;

$<$ Proposition 3.1>

If there exists a perceivable (possible) human action, there should be an affordance and an effectivity that are perceivable, such that $\exists P A \rightarrow \exists P$ and $\exists Q$

Let us now consider the case of existences of an affordance and effectivity. While their existence does not necessitate action, both affordance and effectivity must exist at the same time and place in order for an action to be actualized (Gibson 1979; Turvey 1992). This is the basic assumption of the theory of affordance and also the sufficient condition of existence of a perception-based human action in AES. A sufficient condition means that even though a human does not perceive the properties of AES, the possibility of action exists as long as there is a proper affordance-effectivity dual in the system.

$<$ Proposition 3.2>

If there exists a specific affordance-effectivity dual at the same time $(T)$ and space $(D)$, there should be a possible human action that can be actualized at the time $(T)$ and space $(D)$, such that;

$\exists P$ and $\exists Q \rightarrow \exists P A$, where

$W_{p q}=j\left(X_{p}, Z_{q}\right)$ and, $X$ and $Z$ exist at the same time $(T)$ and space $(D)$.

Based on Proposition 3.1 and Proposition 3.2, we can say that an affordance and an effectivity exist at a specific time and space if and only if there exists a perception-based action. In prospective controls, the possibility of human actions can therefore be assessed based on the existence of human sensory (perceivable) information that composes an affordance-effectivity dual. The affordance and effectivity have to be defined within the same space and time in order to provide opportunities for a human to take a physical action. It should be noted that sensory information that directly affects these higher order properties are categorized as sight, sound, and touch in Figure 3.

$<$ Proposition 3.3>

There exists a perceivable (possible) human action at a specific time (T) and space (D), if and only if there is a specific affordance-effectivity dual at the same time $(T)$ and space $(D)$, which are perceivable, such that;

$\exists P A \leftrightarrow \exists P$ and $\exists Q$, where

$W_{p q}=j\left(X_{p}, Z_{q}\right)$ and, $X$ and $Z$ exist at the same time $(T)$ and space $(D)$. 


\subsection{Representation of Affordances and Effectivities in Systems Modeling}

The concept of perception-based action is the foundation of our modeling formalism, because there are a variety of approaches that can model human activities in systems. There also might be many different cases of human actions such as involuntary or voluntary ones, and expected or unexpected ones. In prospective control, human actions initiated to achieve some goal state should contain proper information to obtain the proper goal(s). Sensible information promotes human actions toward the goal(s) from a set of states in the animal-environment system. We focus on activities in which perception and action are coupled and are not mediated by decision processes for which the same perception may result in multiple different actions.

A necessary-sufficient condition for existing perception-based human actions in prospective controls was presented in the previous section. Based on the necessary-sufficient condition, we can suggest a mathematical representation of affordances and effectivities with respect to actions in systems modeling. The general representation of properties of affordances and effectivities is described as follows;

$$
\begin{aligned}
& \exists P A \leftrightarrow \exists P \text { and } \exists Q \text {, if and only if } \\
& j\left(X_{p}, Z_{q}\right)=W_{p q} \text { and, } X \text { and } Z \text { exist at the same time }(T) \text { and space }(D) \text {, where } \\
& f_{\varphi}: \cup_{i}\left\{\varphi_{i}: W_{p q}\right\} \rightarrow P A, f_{\varphi}: \cup_{i}\left\{\varphi_{i}: X_{p}\right\} \rightarrow P \text {, and } f_{\varphi}: \cup_{i}\left\{\varphi_{i}: Z_{q}\right\} \rightarrow Q \text {. }
\end{aligned}
$$

The mathematical definitions contain dynamic constraints in time and space dimensions among perceivable properties in systems. They can be directly fit into the FSA model introduced in Section 2.1 without increasing the number of variables or tuples. The necessary-sufficient condition and its mathematical representation help us build a logical model and provide a validation tool for checking the existence of dynamic properties of affordances, effectivities, and possible actions.

In the descriptive formalism for the affordance-based FSA model, perceptive physical necessarysufficient conditions were not considered in detail. The consideration of perceptual components and necessary-sufficient conditions leads to the complete model for prospective control.

By combining the necessary-sufficient condition of existence of perception-based actions in prospective control, a mathematical definition of the affordance-based FSA, $\boldsymbol{M}^{\text {comb }}$ (Combined model), that describes the rules of state transitions is developed as follows;

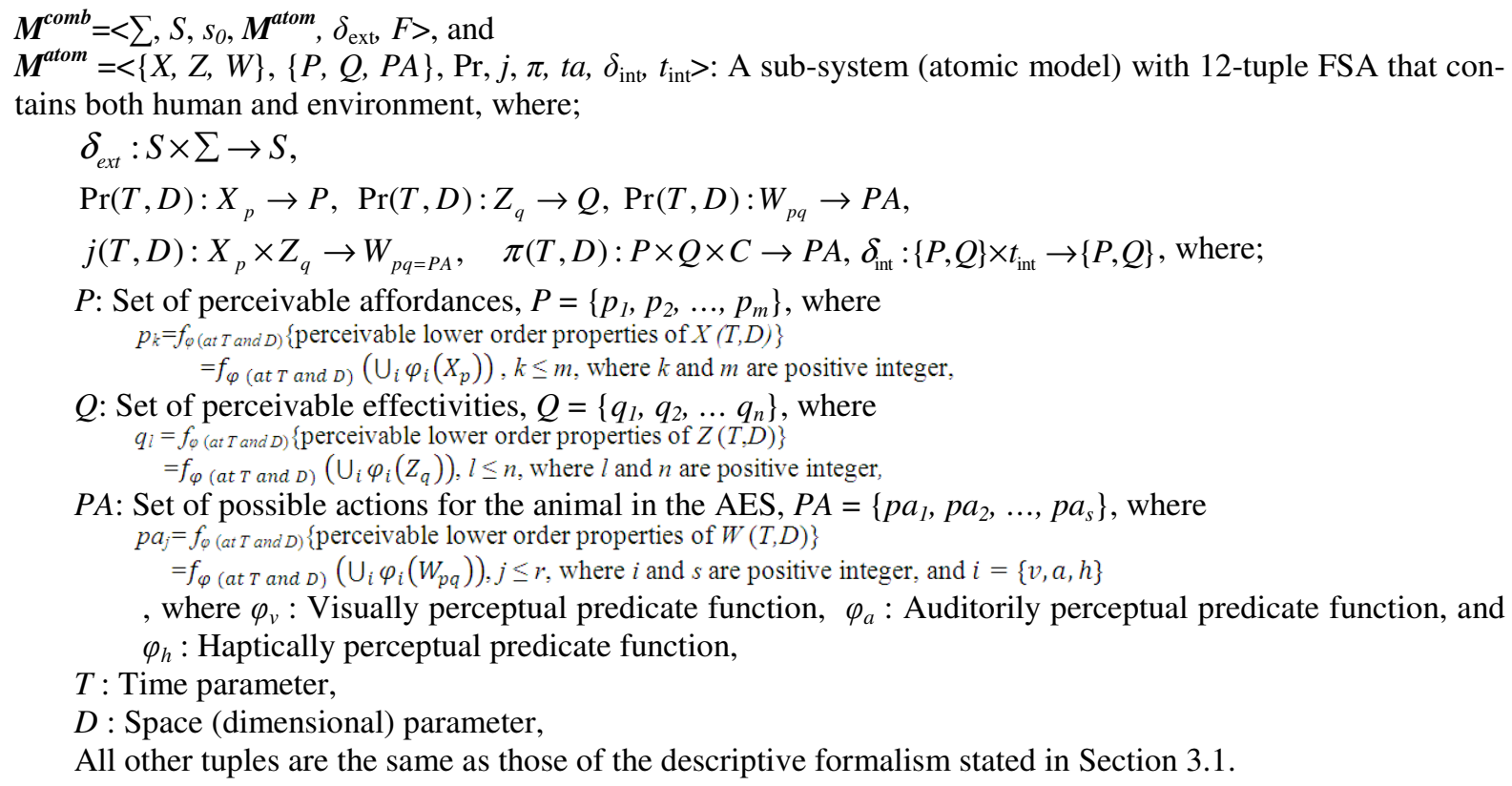




\section{ILLUSTRATIVE EXAMPLE: HIGHWAY-LANE-DRIVER SYSTEM}

Let us consider an example, a Highway-Lane-Driver System (HLDS), that is an animal environment system with two highway lanes and an exit lane as shown in Figure 4 (Thiruvengada and Rothrock 2007). The goal of drivers in this system is to exit the HLDS from their respective lanes by maintaining their target speed without crashing into each other. The driver is considered an 'animal' in the AES. We assume that 1) multiple drivers can share the HLDS, 2) a lane ( $L i)$ within the HLDS provides the affordance "isdrivable" to a driver ( $d j$ ), if and only if, the lane is empty for at least three car lengths (assumed safety factor for moving into lane without creating a problem like an accident) at any given time, 3) the drivers possess the capability to perceive the affordances offered by the environment in a concurrent manner, and would therefore be able to decide whether their adjacent lane provides the affordance "is-drivable" or not, 4) the drivers perceive the environs based on their visual information and they can have 360 degree viewing angle through a front glass, a room mirror, and side mirrors, and 5) the drivers do not accelerate or decelerate during the course of the scenario and maintain their velocity throughout driving.

The goal of drivers in this system is to travel and exit the highway without creating a problem such as an accident and stopping. The goal can be decomposed into tasks and a series of actions associated with the goal. The required actions in this system are 'drive to the L3,' 'drive to the L2,' 'drive to the L1,'and 'drive to the exit.'

For each individual driver, the AES states are extracted based on the set of actions defined above. The states represent the information containing the position of each driver/vehicle and proper combinations between the driver (animal) and the road (environment). We can represent the system states and transitions for the system model, $\boldsymbol{M}^{\text {comb }}$, for each driver as follows;

A set of states: $S=\left\{s_{0}, s_{1}, s_{2}, s_{3}, s_{4}\right\}$, where $s_{0}=$ the starting state representing the driver 1 is in the lane L1, $s_{1}=$ the driver 1 is is driving in the lane L2, $s_{2}=$ the driver 1 is driving in the lane L3, $s_{3}=$ goal state representing the driver 1 exits the highway, and $s_{4}=$ absorbing state (accident or any situation that cannot achieve the goal).

A set of transitions (Human actions) : $\sum=\{$ Drive to L1, Drive to L2, Drive to L3, Exit\}.

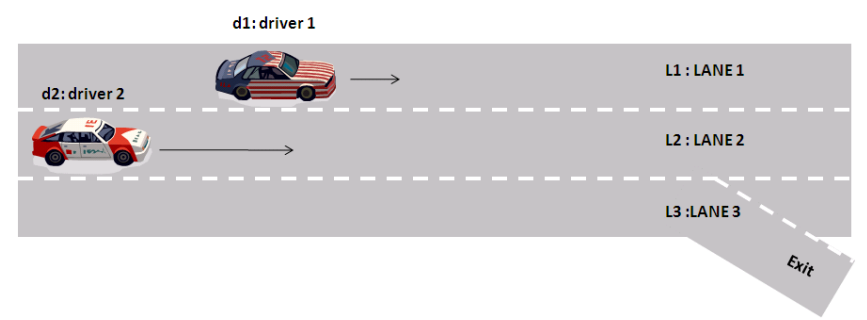

Figure 4: Illustrated example: a Highway-Lane-Driver System (HLDS).

The only thing changed in the modeling representation for each driver is the starting states. The starting states are $s_{0}$ and $s_{l}$ for driver 1 and driver 2, respectively. The system states and transitions among the states for the driver trying to merge right in the example are depicted in Figure 5. Each transition represents a human action to change the current state to the next one in a specific situation. Proper series of transitions lead to the final goal states (attainable or not).

Once specifying all the states and transitions in the AES, perceptual elements in the system need to be identified. To this ends, we create atomic elements (sub states) inside each AES state. For this example, we will consider the case of transitions among states $s_{0}, s_{1}$, and $s_{4}$ as shown in Figure 6 . To make a proper transition to the next state, the human driver must consider the appropriate perceptual conditions of affordance and effectivity states in order to actualize a human action. For example, two different perceptions of situations in $\mathrm{s}_{0}$ are going to be considered. The proper combinations of affordance and effectivity for the transition of 'Drive to L2' between $\mathrm{s}_{0}$ and $\mathrm{s}_{1}$ should be; 
$\left(p_{1}, q_{1}\right)$ and $\left(p_{2}, q_{2}\right)$, where $p_{1}=$ 'perceived drive-ability of LI' and $q_{1}=$ 'driver's perceived capability to make a lane change to or keep going on L1,' $p_{2}=$ 'perceived drive-ability of $L 2$,' and $q_{2}=$ 'driver's perceived capability to make a lane change to or keep going on L2.'

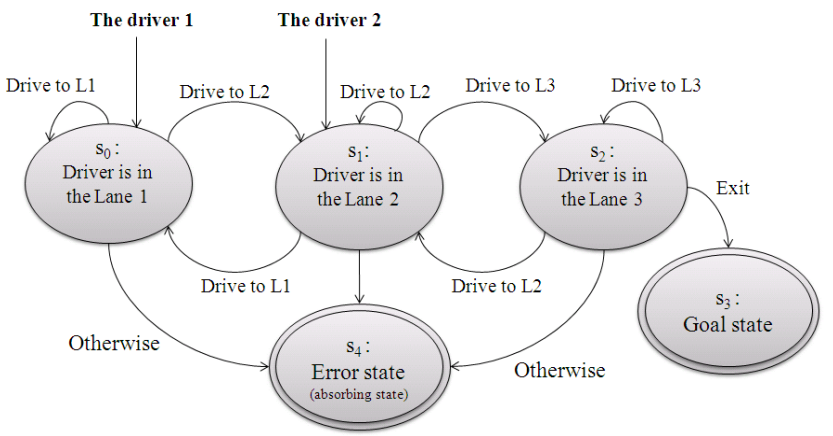

Figure 5: FSA representation of the driving system for each driver/vehicle in a lane.

As defined above, the perceptual information of affordance, effectivity, and possible action are represented as functions of perceptual elements such as dimension, location, and color within a specific time and space range. In this example, the visual information is only considered as perceptual information. The elements that allow the system to make a transition in a dynamic situation can be defined within 'time-space' dimensions as follows;

$$
\begin{aligned}
& \left.p_{1}=f_{\varphi(\mathrm{t} 1, \mathrm{~L} 1)}\left(\varphi_{v}\left(X_{p}\right)\right)=f_{\varphi(\mathrm{t} 1, \mathrm{~L} 1)} \text { (empty lengths of } \mathrm{L} 1\right) \\
& \left.q_{1}=f_{\varphi(\mathrm{t} 1, \mathrm{~L} 1)}\left(\varphi_{v}\left(Z_{p}\right)\right)=f_{\varphi(\mathrm{t} 1, \mathrm{~L} 1)} \text { (velocity of } \mathrm{d} 1 \text {, location of } \mathrm{d} 1 \text {, the length of the car for } \mathrm{d} 1\right) \\
& \left.p_{2}=f_{\varphi(\mathrm{t} 1, \mathrm{~L} 2)}\left(\varphi_{v}\left(X_{p}\right)\right)=f_{\varphi(\mathrm{t} 1, \mathrm{~L} 2)} \text { (empty lengths of } \mathrm{L} 2\right) \\
& q_{2}=f_{\varphi(\mathrm{t} 1, \mathrm{~L} 2)}\left(\varphi_{v}\left(Z_{p}\right)\right)=f_{\varphi(\mathrm{t} 1, \mathrm{~L} 2)}(\text { velocity of } \mathrm{d} 1 \text {, location of } \mathrm{d} 1 \text {, the length of the car for } \mathrm{d} 1) \\
& \text {, where } \\
& f_{\varphi}: \text { A function of organization and interpretation in a perceptual process, and } \\
& \mathrm{t}_{1}: \text { The moment that the driver d1 perceives the system. }
\end{aligned}
$$

The internal and external transitions are represented as the connection between specific combinations of affordance-effectivity states, and a set of possible action is generated by a specific affordanceeffectivity combination as follows:

$$
\begin{aligned}
& \delta_{\text {int }}\left(\left(p_{*}, q_{*}\right), t_{\text {int }}\right)=\left(p_{2}, q_{2}\right) \text {, and } \\
& \pi_{(t 1, L 1)}\left(p_{2}, q_{2}, c_{2}\right)=p a_{2}={ }^{\prime} \text { Drive to L2 under } c_{2}{ }^{\prime} \text {, where } \\
& c_{2}=\{\mathrm{L} 2 \text { is empty for at least three car lengths, d1 did not pass by the exit }\} \\
& \text { Thus, } \delta_{\text {ext }}\left(s_{0},{ }^{\prime} \text { Drive to L2 under } c_{2}{ }^{\prime}\right)=\delta_{\text {ext }}\left(\left(p_{1}, q_{1}\right),{ }^{\prime} \text { Drive to L2 under } c_{2}{ }^{\prime}\right)=s_{1} .
\end{aligned}
$$

We can develop an FSA model by using a similar approach to that described above for all AESs as shown in Figure 6 where other affordances and effectivities are defined as follows;

$p_{3}=$ 'perceived drive-ability of $L 3$ ' $=f_{\varphi \text { (current time, lane) }}$ (empty lengths of $L 3$ ),

$q_{3}=$ 'driver's perceived capability to make a lane change to or keep going on $L 3$ '

$=f_{\varphi}$ (current time, lane $)($ velocity of $\mathrm{d} 1$, location of $\mathrm{d} 1$, the car length of $d 1)$,

$p_{4}=$ 'perceived drive-ability of the exit' $=f_{\varphi \text { (current time,lane) }}($ empty lengths of $L 3$ ), and

$q_{4}=$ 'driver's perceived capability to drive to the exit'

$=f_{\varphi(\text { current time, lane })}($ velocity of $\mathrm{d} 1$, location of $\mathrm{d} 1$, the car length of $d 1)$.

The above affordances and effectivities are expressed as functions of the lower order visual properties such as empty lengths on lanes, velocity of cars, and position of drivers. The existence of a possible action depends on the necessary-sufficient condition of the existence of a certain action within dynamically perceivable in the time and space dimensions. Between system states, we can also deploy required physical conditions (assumptions) for realizing actions as follows; 
$c_{1}=\{L 1$ is empty for at least three car lengths, $d 1$ did not pass by the exit $\}$

$c_{3}=\{L 3$ is empty for at least three car lengths, $d 1$ did not pass by the exit $\}$

$c_{4}=\{$ the exit is empty, $d l$ did not pass by the exit $\}$

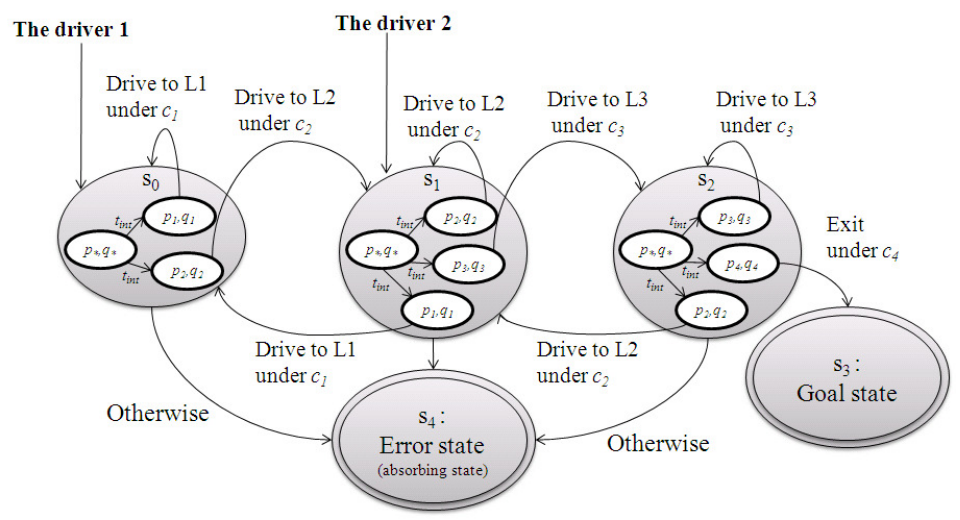

Figure 6: The affordance-based FSA model for each driver/vehicle of the driving systems in Figure 4.

The properties of an affordance and effectivity need to be expressed into lower level perceptual properties such as dimension, velocity, and position. When modeled properly, the generated FSA model can work in a dynamic environment and the properties in models are predictable as long as we can evaluate what a driver perceives though his or her own sensory (vision, hearing, etc.) systems.

The evaluation of human perceptual information is provided in the model as an input and the state transition occurs based on the dynamic changes of perceptual properties (lower order properties consisting affordances and effectivities). The satisfaction of the necessary-sufficient condition of a perceptionbased human action at each state guarantees the existence of transitions to the next system states (reachability to the next goal states). If not, the system goes directly to the absorbing state $\left(\mathrm{s}_{4}\right)$.

\section{CONCLUSIONS}

In this paper, we have explored the soundness and completeness of an affordance-based modeling formalism in order to develop computable models for prospective control of human actions in a complex system. Unlike a machine, humans take an action based on perceptual information in nondeterministic and autonomous ways. The perception coupled with the environment states contributes to human actions and it can be used to predict human effects on these systems. In this sense, the perceptual properties of affordance and effectivity in the system should be considered in system representation with their related actions.

In regard of prescriptive evaluations for perception-based human actions we investigate the dynamic perceptual properties in human actions and propose the following necessary-sufficient conditions for the generation of perception-based human actions: 1) A person should be able to perceive a set of affordanceeffectivity-possible actions, which can be interpreted as a set of lower order properties through his or her perceptual organs, and 2) In each AES state, a person and an environmental element should exist at the same time and space dimension.

The affordance-based FSA model generated based on the perceptual properties of perception-based human actions and the definitions of necessary-sufficient conditions can play a role as a basic framework for decomposing goals and tasks in human-involved systems. In the atomic level modeling, the perceptual elements of affordances, effectivities, and possible actions are defined within dynamic perceptual constraints that coincide with the necessary-sufficient conditions. This model can be generalized to accom- 
modate domain-independence within various human-involved systems modeling and control. We illustrated these procedures with a simple example of the highway-lane-driver system.

We note that it might be difficult to measure or evaluate the system affordances associated with a human in a quantitative way in some cases. However, once we analyze them, the evaluation of affordances and effectivities will help us understand and predict human actions in complex environs. The controller developed by the affordance-FSA formalism can work with both system controllers and simulation software enabling us to develop a general framework for human-machine or human-computer cooperative systems. The proposed formal models provide the breadth and depth for hierarchical systems such as a supply chain model, which contains complexity along with a variety of human activities.

\section{REFERENCES}

Arkin, R.C. 1998. Behavior-based robotics. Cambridge, MA: MIT Press.

Fajen, B.R. 2005. Perceiving possibilities for action: On the necessity of calibration and perceptual learning for the visual guidance of action. Perception 34: 717-740.

Gibson, J.J. 1966. In the senses considered as perceptual systems. Boston, MA: Houghton Mifflin.

Gibson, J.J. 1979. The ecological approach to visual perception. Boston, MA: Houghton Mifflin.

Gibson, J.J. 1982. Reasons for realism: Selected essays of james j. Gibson. Hillsdale, NJ: Lawrence Erlbaum.

Huffman, K. 2007. Psychology in action. 8th ed. New York, NY: John Wiley \& Sons Inc.

Jones, K.S. 2003. What is an affordance. Ecological Psychology, 15(2): 107-114.

Kim, N., H. Thiruvengada, L. Rothrock, R. A. Wysk, and D. Shin. 2008. Modeling of affordances in human-involved complex systems using finite state automata (FSA). IEEE Transactions on Systems, Man, and Cybernetics- Part A: Humans and Systems (In revision).

Kim, N., D. Shin, R. A. Wysk, and L. Rothrock. 2010. Using Finite State Automata (FSA) for the Formal Modeling of Affordances in Human-Machine Cooperative Systems. International Journal of Production Research. 48(5): 1303-1020.

Kirlik, A., R. A. Miller, and R. J. Jagacinski. 1993. Supervisory control in a dynamic uncertain environment: A process model of skilled human-environment interaction. IEEE Transactions on Systems, Man, and Cybernetics, 23(4): 929-952.

Norman, D. 1988. The psychology of everyday things. New York, NY: Basic Books.

Levine, M.W. 2000. Levine\&Shefner's Fundamentals of Sensation and Perception. New York, NY: Oxford University Press.

Pirolli, P. and S. Card. 1999. Information foraging. Psychological Review. 106(4) : 643-675.

Shaw, R.E. 2003. The agent-environment interface: Simon's indirect or Gibson's direct coupling? Ecological Psychology. 15(1): 37-106.

Shaw, R.E. and Turvey, M.T. 1981. Coalitions as models for ecosystems: A realist perspective on perceptual organization. In M. KubovyandPomerantz, J.R. eds. Perceptual organization. Hillsdale, NJ: Lawrence Erlbaum Associates, Inc.: 343-415.

Simon, H.A. 1996. The sciences of the artificial. 3rd ed., Cambridge, MA: MIT Press (Original work published in 1969).

Thiruvengada, H. and L. Rothrock. 2006. HCI implications of a colored petri net model for gibson's affordance. In Proceedings of the 2006 Industrial Engineering Research Conference. Orlando, FL, May 2006: Institute of Industrial Engineers.

Thiruvengada, H. and L. Rothrock. 2007. Affordance-based Computational Model of Driver Behavior on Highway Systems: A Colored Petri Net Approach. In Proceedings of the 2007 IEEE International Conference on Systems, Man and Cybernetics. Montreal, QC, Canada, Oct. 2007: Institute of Electrical and Electronics Engineers, Inc.

Turvey, M. T. 1992. Affordances and prospective control: An outline of the ontology. Ecological Psychology. 4: 173-187. 


\section{AUTHOR BIOGRAPHIES}

NAMHUN KIM is an Assistant Professor at Design and Human Engineering, Ulsan National Institute of Science and Technology in South Korea. He received the B.S. and M.S. degrees in mechanical engineering from Korea Advanced Institute of Science and Technology (KAIST) in South Korea, in 1998 and 2000, respectively, and the Ph.D. degree in industrial engineering from the Pennsylvania State University, University Park in 2010. His research interests are in human-machine systems, human-involved systems modeling and controls, agent-based simulation, and information system designs.

JAEKOO JOO is a Professor of Systems and Management Engineering Department, Inje University in South Korea. He received the B.S. degrees in industrial engineering from the Seoul National University, South Korea in 1987 and the M.S. and Ph.D. degree in industrial engineering from POSTECH, South Korea in 1997 and 2000 respectively. His research interests include modeling and simulation for human involved system, production management, and lean manufacturing. Dr. Joo is a member of the Institute of Industrial Engineers.

LING ROTHROCK is an Associate Professor in the Harold and Inge Marcus Department of Industrial and Manufacturing Engineering, Pennsylvania State University, University Park. He received the Ph.D. degree in industrial engineering from the Georgia Institute of Technology, Atlanta, in 1995. He served as an Officer in the U.S. Army until 2000. His research interests include human-in-the-loop discrete event simulations, display visualization, and human-machine performance assessment. His simulation and modeling software have been used by the U.S. Navy and the Army for training and interface design. He has published over 50 technical articles in the area. Dr. Rothrock is a senior member of the IEEE, a member of the Institute of Industrial Engineers and the Human Factors and Ergonomics Society.

RICHARD A. WYSK is the Dopaco Distinguished Professor of industrial and systems engineering at the North Carolina State University and the emeritus professor at the Pennsylvania State University. Before joining NCSU, he was the William E. Leonhard Chair in Engineering and a Professor of Industrial Engineering at the Pennsylvania State University, University Park. He received the B.S. and M.S. degrees in industrial engineering and operations research from the University of Massachusetts, Amherst, in 1972 and 1973, respectively, and the Ph.D. degree in industrial engineering from Purdue University, West Lafayette, IN, in 1977. His research and teaching interests are in lean manufacturing, computer-aided process planning, and flexible manufacturing systems (FMSs) planning, design, and control. He has coauthored six books. He has also published more than 150 technical papers in the open literature in journals including the Transactions of ASME, the Transactions of IEEE, and the IIE Transactions. Dr. Wysk is an IIE Fellow, a Fellow of SME, a member of Sigma Xi, and a member of Alpha Pi Mu and Tau Beta Pi. He serves as the Chair of the SME Journals Committee and is an Associate Editor for six engineering journals. 